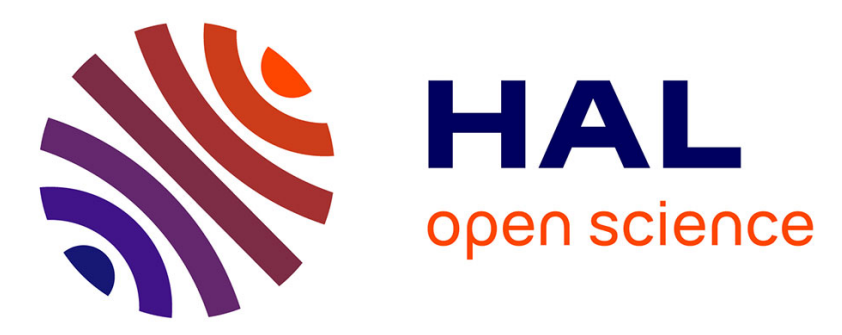

\title{
Energy-density field approach for low- and medium-frequency vibroacoustic analysis of complex structures using a statistical computational model
}

\author{
M. Kassem, Christian Soize, L. Gagliardini
}

\section{- To cite this version:}

M. Kassem, Christian Soize, L. Gagliardini. Energy-density field approach for low- and mediumfrequency vibroacoustic analysis of complex structures using a statistical computational model. Journal of Sound and Vibration, 2009, 323 (3-5), pp.849-863. 10.1016/j.jsv.2009.01.014 . hal-00684343

\section{HAL Id: hal-00684343 \\ https://hal.science/hal-00684343}

Submitted on 1 Apr 2012

HAL is a multi-disciplinary open access archive for the deposit and dissemination of scientific research documents, whether they are published or not. The documents may come from teaching and research institutions in France or abroad, or from public or private research centers.
L'archive ouverte pluridisciplinaire HAL, est destinée au dépôt et à la diffusion de documents scientifiques de niveau recherche, publiés ou non, émanant des établissements d'enseignement et de recherche français ou étrangers, des laboratoires publics ou privés. 


\title{
Energy density field approach for low- and medium-frequency vibroacoustic analysis of complex structures using a statistical computational model.
}

\author{
M. Kassem ${ }^{\text {a,b }}$, C. Soize ${ }^{a, *}$, L. Gagliardini ${ }^{b}$ \\ ${ }^{a}$ Université Paris-Est, Laboratoire de Modélisation et Simulation Multi Echelle, \\ MSME FRE3160 CNRS, 5 Bd Descartes,77454 Marne-la-Vallee, France \\ ${ }^{\mathrm{b}}$ PSA Peugeot Citroen Route de Gisy, 78943 Velizy-Villacoublay, France
}

\begin{abstract}
In this paper, an energy-density field approach applied to the vibroacoustic analysis of complex industrial structures in the low- and medium-frequency ranges is presented. This approach uses a statistical computational model. The analyzed system consists of an automotive vehicle structure coupled with its internal acoustic cavity. The objective of this paper is to make use of the statistical properties of the frequency response functions of the vibroacoustic system observed from previous experimental and numerical work. The frequency response functions are expressed in terms of a dimensionless matrix which is estimated using the proposed energy approach. Using this dimensionless matrix, a simplified vibroacoustic model is proposed.
\end{abstract}

Key words: Vibroacoustics, Energy Analysis, Stochastic computational Model, Uncertainties.

\section{Introduction}

Industrial computational models developed for the vibroacoustic analysis of complex structures in the low- and medium-frequency ranges, are mainly constructed using the finite element method which enables complex systems to

* Corresponding author
Email address: christian.soize@univ-paris-est.fr (C. Soize). 
be analyzed. However, even if the predictability of these models is quite acceptable for general conception purposes, there still exists a gap between experimental analysis and numerical analysis. This gap is due to uncertainties existing not only in the parameters of the physical system and their measuring procedures, but also in the numerical model itself. This is why applying statistical methods becomes necessary to compensate for those uncertainties. One well known statistical approach is the parametric probabilistic approach. This approach takes into account uncertainties in the physical system parameters but does not take into account the computational model uncertainties. To take into account these model uncertainties, the non-parametric probabilistic approach of model uncertainties presented in [1] [2] [3] is used in this work to construct a statistical computational model.

Statistical methods based on energy analysis like the well known Statistical Energy Analysis (SEA) [4][5] are devoted to the high-frequency range where the number of modes is very high and the statistical properties are quite evident. On the other hand in the low- and medium-frequency ranges, global modes and local modes are present simultaneously in a same narrow frequency band. The SEA and its derivations have been developed and implemented in a lot of previous research works such as those presented in [6][7][8][9][10] [11][12][13]. The efficiency of those methods in the high-frequency range is due to the small statistical fluctuations with respect to the mean values obtained using frequency averaging. This is not the case in the low- and mediumfrequency ranges where the mean values are no more representative of the response due to very high statistical fluctuations. In addition, in these frequency ranges, we need to keep the mean phases of the responses which are not linearly decreasing. It is well understood today that only a computational model derived from the complete boundary value problem is necessary. Another reason why statistical methods using frequency averaging are not adapted to low- and medium- frequency ranges is that such averaging induces a loss of the frequency resolution which is not compatible with the frequency responses in these frequency ranges for lightly damped systems. For this last reason frequency averaging is sometimes replaced by spatial averaging. This type of averaging is very difficult to implement on complex structures like automotive vehicles structures and may be problematic in these cases. Consequently, the most appropriate strategy is to use an ensemble averaging applied to a family of random systems requiring the explicit construction of the probability model. This strategy is adopted in the present work by using a probabilistic computational model constructed using the non-parametric probabilistic approach. Using the ensemble averaging, statistical properties of the response that have been observed in previous work [14] [15] in the low- and medium frequency ranges in a stochastic context can be exploited.

It should be noted that references [14] and [15] deal with the prediction and 
experimental validation in the low- and medium-frequency ranges of the complex vibroacoustic systems, such as an automotive vehicle, using an uncertain computational computational model. In this work, the random Frequency Response Functions (FRF) are predicted for all the frequencies in the band of interest and with a spatial resolution corresponding to the used finite element mesh. Such complex systems can have several millions of degrees of freedom, and consequently, several millions of FRF. The objective of the present work is to significantly reduce the number of FRF, constructed by the method proposed in references [14] and [15], through replacing several millions of FRF by only a few hundred FRF while keeping a reasonable accuracy of the vibroacoustic responses.

A simplified vibroacoustic model for low- and medium-frequency bands is proposed based on an energy-density field approach which uses the statistical computational model. This approach requires a full stochastic vibroacoustic analysis using the non-parametric probabilistic approach of uncertainties for which the CPU time is denoted by $\tau_{\text {full }}$. A post-treatment of the results of this full stochastic analysis is then needed to construct the simplified vibroacoustic model. The CPU time required for this post-treatment is denoted as $\Delta \tau_{\text {simpl }}$. For a very large computational vibroacoustic model, $\Delta \tau_{\text {simpl }}$ is not significant with respect to $\tau_{\text {full }}$ which means that the total CPU time is equivalent to $\tau_{\text {full }}$. Consequently, there is no additional CPU time with respect to the full stochastic vibroacoustic computation. It should be noted that the introduction of such simplified vibroacoustic model is not carried out to decrease the CPU time, but is rather developed to help save time in the phase of the design process by engineers. Compared to CPU time, this gaining of time is much more significant.

The stochastic reduced computational model of the vibroacoustic system is obtained from the mean reduced computational model using the non-parametric probabilistic approach of both model uncertainties and system parameters uncertainties. The stochastic reduced matrix equation is then solved using the Monte Carlo method. The main idea of the proposed energy approach is based on a normalization of the FRF using both the input and the output mobilities of the system. The vibroacoustic energy analysis is performed in a local coordinate system to ensure better analysis of the structural deformations. The reason of using these local coordinates will be explained in details later.

It should be noted that, the normalization of the FRF with respect to the input and output mobilities has been presented in previous works dedicated to energy methods. However, the mobilities used in these works are not the same as those used in the present work. For example reference [16] uses what is called the energy mobility. Moreover, those methods use either frequency or spatial averaging. 
For shortness the mean vibroacoustic model is not presented in this paper. The mean reduced model and the stochastic vibroacoustic model are briefly summarized. The reader is referred to [17] for the general formulation of the mean model and to [14] and [15] for the stochastic formulation in the context of automotive structures.

The general structure of this paper is then presented as follows: First, we present the mean reduced computational model of the vibroacoustic system. Second, the application of the non-parametric probabilistic approach of uncertainties on the mean reduced model is presented. The Energy density field approach and the transformation from the global coordinate system to the local one are then explained. Then, we present the construction of the simplified vibroacoustic model. Finally, the results and the conclusion are presented in the last two sections.

\section{Notation}

The following notations are used in this paper:

- Lower case bold letters stand for deterministic vectors (e.g. u).

- Curved capital letters stand for the mean reduced model deterministic matrices (e.g. $\mathcal{A})$.

- $\mathbb{f}$ is the force vector of the mean reduced model.

- Bold upper case letters stand for random matrices of the stochastic computational model (e.g. A).

- Any underlined quantity means that this is the mean statistical value of this quantity (e.g. T).

- The superscripts " $s$ " stands for the structure and " $f$ " stands for the acoustic cavity (e.g. $\mathbf{q}^{s}$ and $\mathbf{q}^{f}$ ).

- The superscript "loc" stands for a quantity in the local coordinates (e.g. $\left.\mathcal{E}^{l o c}\right)$.

- The superscript $\ell$ indicates directions of the local coordinates system $\left(X^{\ell}, Y^{\ell}\right.$, $\left.Z^{\ell}\right)$.

\section{Reduced mean computational vibroacoustic model}

For all angular frequencies $\omega$ belonging to the frequency band of analysis $B=$ $\left[\omega_{\text {min }}, \omega_{\text {max }}\right]$ with $\omega_{\min }>0$, the reduced mean computational vibroacoustic 
model is written as

$$
\mathbf{u}^{s}(\omega)=\Psi \mathbf{q}^{s}(\omega) \quad, \quad \mathbf{p}^{f}(\omega)=\Phi \mathbf{q}^{f}(\omega),
$$

in which $\mathbf{q}^{s}(\omega)$ is the vector of the generalized structural coordinates, with values belonging to $\mathbb{C}^{n}$, associated with the $n$ first structural elastic modes constituting the matrix $\Psi$ and in which $\mathbf{q}^{f}(\omega)$ is the vector of the generalized acoustical coordinates, with values belonging to $\mathbb{C}^{m}$, associated with the $m$ first acoustic modes constituting the matrix $\Phi$ which includes the constant pressure mode at zero eigenfrequency. $\mathbf{q}^{s}(\omega)$ and $\mathbf{q}^{f}(\omega)$ verify the matrix equation

$$
\left[\begin{array}{cc}
\mathcal{A}^{s}(\omega) & \mathcal{C} \\
\omega^{2} \mathcal{C}^{T} & \mathcal{A}^{f}(\omega)
\end{array}\right]\left[\begin{array}{l}
\mathbf{q}^{s}(\omega) \\
\mathbf{q}^{f}(\omega)
\end{array}\right]=\left[\begin{array}{l}
\mathbb{F}^{s}(\omega) \\
\mathbb{f}^{f}(\omega)
\end{array}\right] .
$$

In Eq. (1), $\mathbf{u}^{s}(\omega)$ and $\mathbf{p}^{f}(\omega)$ are, the vector of the structural DOF's with values belonging to $\mathbb{C}^{n_{s}}$, and the vector of the acoustical DOF's with values belonging to $\mathbb{C}^{n_{f}}$, respectively. In Eq. (2), $\mathcal{A}^{s}(\omega)$ and $\mathcal{A}^{f}(\omega)$ are, respectively, the generalized dynamical stiffness matrix of the structure and the generalized dynamical stiffness matrix of the acoustic cavity which are defined by

$$
\begin{aligned}
& \mathcal{A}^{s}(\omega)=-\omega^{2} \mathcal{M}_{n}^{s}+i \omega \mathcal{D}_{n}^{s}+\mathcal{K}_{n}^{s}, \\
& \mathcal{A}^{f}(\omega)=-\omega^{2} \mathcal{M}_{m}^{f}+i \omega \mathcal{D}_{m}^{f}+\mathcal{K}_{m}^{f}
\end{aligned}
$$

In Eq. (3), $\mathcal{M}_{n}^{s}, \mathcal{D}_{n}^{s}$ and $\mathcal{K}_{n}^{s}$ are positive-definite symmetric real $(n \times n)$ matrices corresponding to the generalized mass, damping and stiffness matrices. In Eq. (4) devoted to the acoustic cavity, $\mathcal{M}_{m}^{f}$ is a positive-definite symmetric real $(m \times m)$ matrix corresponding to the generalized "mass" matrix and, $\mathcal{D}_{m}^{f}$ and $\mathcal{K}_{m}^{f}$ are the positive symmetric real $(m \times m)$ matrices corresponding to the generalized "damping" and "stiffness" matrices. Finally, in Eq. (2), $\mathcal{C}$ is the real $(n \times m)$ matrix corresponding to the generalized vibroacoustic coupling matrix and where $\mathbb{E}^{s}(\omega)$ and $\mathbb{E}^{f}(\omega)$ are the generalized structural forces and the generalized acoustical sources applied to the vibroacoustic system.

\section{Stochastic computational vibroacoustic model}

In this work, the non-parametric probabilistic approach [1][2][3] is used to construct the statistical computational vibroacoustic model in order to take into account both parameter and model uncertainties. One refers the reader to [14] for the details of this implementation. In such an approach, the matrices of the reduced mean computational vibroacoustic model are replaced by random matrices whose mean values are equal, by construction, to the matrices of the reduced mean computational vibroacoustic model. Consequently, Eqs. (1) and 
(2) are replaced by the following random equations

$$
\mathbf{U}^{s}(\omega)=\Psi \mathbf{Q}^{s}(\omega) \quad, \quad \mathbf{P}^{f}(\omega)=\Phi \mathbf{Q}^{f}(\omega),
$$

in which, the random vector $\mathbf{Q}^{s}(\omega)$ with values belonging to $\mathbb{C}^{n}$ and the random vector $\mathbf{Q}^{f}(\omega)$ with values belonging to $\mathbb{C}^{m}$, verify the random matrix equation

$$
\left[\begin{array}{cc}
\mathbf{A}^{s}(\omega) & \mathbf{C} \\
\omega^{2} \mathbf{C}^{T} & \mathbf{A}^{f}(\omega)
\end{array}\right]\left[\begin{array}{l}
\mathbf{Q}^{s}(\omega) \\
\mathbf{Q}^{f}(\omega)
\end{array}\right]=\left[\begin{array}{l}
\mathbb{f}^{s}(\omega) \\
\mathbb{f}^{f}(\omega)
\end{array}\right],
$$

where the random matrices $\mathbf{A}^{s}(\omega)$ and $\mathbf{A}^{f}(\omega)$ are written as

$$
\begin{aligned}
& \mathbf{A}^{s}(\omega)=-\omega^{2} \mathbf{M}_{n}^{s}+i \omega \mathbf{D}_{n}^{s}+\mathbf{K}_{n}^{s} \\
& \mathbf{A}^{f}(\omega)=-\omega^{2} \mathbf{M}_{m}^{f}+i \omega \mathbf{D}_{m}^{f}+\mathbf{K}_{m}^{f}
\end{aligned}
$$

In Eq. (7), $\mathbf{M}_{n}^{s}, \mathbf{D}_{n}^{s}$ and $\mathbf{K}_{n}^{s}$ are random matrices with values in the set of all the positive-definite symmetric real $(n \times n)$ matrices. In Eq. (8), $\mathbf{M}_{m}^{f}$ is a random matrix with values in the set of all the positive-definite symmetric real $(m \times m)$ matrices and, $\mathbf{D}_{m}^{f}$ and $\mathbf{K}_{m}^{f}$ are random matrices with values in the set of all the positive symmetric real $(m \times m)$ matrices. Finally, in Eq. (6), $\mathbf{C}$ is a random matrix with values in the set of all the real $(n \times m)$ matrices. The probability distributions of these seven random matrices are completely defined in the nonparametric probabilistic approach and a numerical procedure for generating independent realizations of these random matrices is explicitly known (see [1][2][3]). It should be noted that, in this random matrix theory, the statistical fluctuation level of each random matrix is controlled by a dispersion parameter $\delta>0$. If $\delta=0$ (deterministic case) the random matrix is equal to its mean value. The higher the value of $\delta$, the higher is the uncertainty level.

\section{Implementation of the energy density field approach}

Let $n^{s f}=n^{s}+n^{f}$ be the total number of DOF's. One will only use the subset $\left\{j_{1}, \ldots, j_{\alpha}, \ldots, j_{r}\right\}$ of the $r$ observed DOF's which is equal to the number of excited DOF's for the vibroacoustic system. In general, $r \ll n^{s f}$. Note that the excited DOF's are the same as the observed DOF's. The excitations are represented by external mechanical forces applied to the structure and/or by external acoustic sources in the acoustic cavity. For $\alpha$ fixed in $\{1, \ldots, r\}$, let $t \mapsto \mathbf{f}^{\alpha}(t)$ be the function from $\mathbb{R}$ into $\mathbb{R}^{r}$ representing the excitation vector relative to the DOF's $j_{\alpha}$ which is written as $\mathbf{f}^{\alpha}(t)=\left\{0, \ldots, f_{\alpha}^{\alpha}(t), \ldots, 0\right\}$ and which is such that $\mathbf{f}^{\alpha}(-t)=\mathbf{f}^{\alpha}(t)$. It is assumed that $\mathbf{f}^{\alpha}$ is square integrable on $\mathbb{R}$. Let $\mathbf{f}^{\alpha}(\omega)=\int_{\mathbb{R}} e^{-i \omega t} \mathbf{f}^{\alpha}(t) d t$ be its Fourier transform which is real function such that $\mathbf{f}^{\alpha}(-\omega)=\mathbf{f}^{\alpha}(\omega)$. Consequently, we have $\mathbf{f}^{\alpha}(\omega)=$ $\left\{0, \ldots, f_{\alpha}^{\alpha}(\omega), \ldots, 0\right\}$. Finally, it is assumed that the support of $\omega \mapsto \mathbf{f}^{\alpha}(\omega)$ is 
the bounded interval $\bar{B} \cup B$ in which $\bar{B}=\left[-\omega_{\max },-\omega_{\min }\right]$. Let $\mathbb{Z}(\omega)$ be the $\left(n^{s f} \times n^{s f}\right)$ complex random matrix such that

$$
\mathbb{Z}(\omega)=\left[\begin{array}{ll}
\Psi & 0 \\
0 & \Phi
\end{array}\right]\left[\begin{array}{cc}
\mathbf{A}^{s}(\omega) & \mathbf{C} \\
\omega^{2} \mathbf{C}^{T} & \mathbf{A}^{f}(\omega)
\end{array}\right]^{-1}\left[\begin{array}{cc}
\Psi^{T} & 0 \\
0 & \Phi^{T}
\end{array}\right],
$$

which exists for all $\omega$ in $B$. Let $\mathbf{Z}(\omega)$ be the $(r \times r)$ complex random matrix such that, for all $\alpha$ and $\beta$ in $\{1, \ldots, r\}$, one has

$$
\mathbf{Z}_{\alpha \beta}(\omega)=\mathbb{Z}_{j_{\alpha} j_{\beta}}(\omega)
$$

For all $\omega$ fixed in $B$, let $\mathbf{T}(\omega)$ be the $(r \times r)$ complex random matrix defined by

$$
\mathbf{T}(\omega)=i \omega \mathbf{Z}(\omega)
$$

The function $\omega \mapsto \mathbf{T}(\omega)$ is called the matrix-valued random FRF related to the excited and to the observed DOF's. It should be noted that $\mathbf{T}(-\omega)=\overline{\mathbf{T}(\omega)}$. For $\alpha$ fixed in $\{1, \ldots r\}$, let $\mathbf{V}^{\alpha}(\omega)$ be the complex random vector of the velocity responses for the observed DOF's $\left\{j_{1}, \ldots, j_{r}\right\}$. One then has

$$
\mathbf{V}^{\alpha}(\omega)=\mathbf{T}(\omega) \mathbf{f}^{\alpha}(\omega)
$$

We now introduce the $(r \times r)$ random mobility matrix $\mathbf{Y}(\omega)$ of the vibroacoustic system for the excited and for the observed DOF's. Below one uses the terminology introduced in references [18] [19] concerning the driving point mobility functions and the coupling mobility functions. . In the present work we are only interested in the driving point mobility functions and not in the coupling mobility functions, the random mobility matrix is a $(r \times r)$ real diagonal random matrix defined by

$$
\mathbf{Y}_{\alpha \beta}(\omega)=\left\{\begin{array}{cc}
\operatorname{Re}\left(\mathbf{T}_{\alpha \alpha}(\omega)\right) & \text { if } \alpha=\beta \\
0 & \text { if } \alpha \neq \beta
\end{array} .\right.
$$

It should be noted that, for all $\omega \in B, \mathbf{Y}_{\alpha \alpha}(\omega)$ is positive-valued random variable which is such that $\mathbf{Y}_{\alpha \alpha}(-\omega)=\mathbf{Y}_{\alpha \alpha}(\omega)$.

The random input power of the vibroacoustic system induced by the excitation $\mathbf{f}^{\alpha}$ is defined by

$$
\boldsymbol{\Pi}_{i n}^{\alpha}=\int_{\mathbb{R}} \mathbf{f}^{\alpha}(t)^{T} \mathbf{V}^{\alpha}(t) d t
$$

Since $\mathbf{f}^{\alpha}(-\omega)=\mathbf{f}^{\alpha}(\omega)$ and since $\mathbf{V}^{\alpha}(-\omega)=\overline{\mathbf{V}^{\alpha}(\omega)}$, one can write

$$
\boldsymbol{\Pi}_{i n}^{\alpha}=\frac{1}{\pi} \int_{B} \mathbf{f}^{\alpha}(\omega)^{T} \operatorname{Re}\left\{\mathbf{V}^{\alpha}(\omega)\right\} d \omega
$$

Substituting Eq. (12) into Eq. (15) yields

$$
\boldsymbol{\Pi}_{i n}^{\alpha}=\frac{1}{\pi} \int_{B} \mathbf{f}^{\alpha}(\omega)^{T} \operatorname{Re}\{\mathbf{T}(\omega)\} \mathbf{f}^{\alpha}(\omega) d \omega,
$$


which can be rewritten as

$$
\begin{aligned}
\mathbf{\Pi}_{i n}^{\alpha} & =\int_{B} \pi_{i n}^{\alpha}(\omega) d \omega \\
\pi_{i n}^{\alpha}(\omega) & =\frac{1}{\pi} \mathbf{f}^{\alpha}(\omega)^{T} \operatorname{Re}\{\mathbf{T}(\omega)\} \mathbf{f}^{\alpha}(\omega)=\frac{1}{\pi} \operatorname{Re}\left\{\mathbf{T}_{\alpha \alpha}(\omega)\right\} f_{\alpha}^{\alpha}(\omega)^{2} .
\end{aligned}
$$

One introduces the vector-valued spectral density function $\mathbf{s}^{f}(\omega)=\left(s_{1}^{f}(\omega), \ldots\right.$, $\left.s_{r}^{f}(\omega)\right)$ belonging to $\left(\mathbb{R}^{+}\right)^{r}$ relative to all the excited DOF's such that

$$
s_{\alpha}^{f}(\omega)=(1 / 2 \pi) f_{\alpha}^{\alpha}(\omega)^{2} .
$$

Similarly, one introduces the random input power density function $\boldsymbol{\pi}_{i n}$ with values belonging to $\left(\mathbb{R}^{+}\right)^{r}$ such that

$$
\boldsymbol{\pi}_{i n}=\left(\pi_{i n}^{1}, \ldots, \pi_{i n}^{\alpha}, \ldots, \pi_{i n}^{r}\right)
$$

which can then be written, using the diagonal matrix $\mathbf{Y}(\omega)$, as

$$
\boldsymbol{\pi}_{i n}(\omega)=2 \mathbf{Y}(\omega) \mathbf{s}^{f}(\omega)
$$

From Eq. (21), it can be deduced that

$$
\mathbf{s}^{f}(\omega)=\frac{1}{2} \mathbf{Y}(\omega)^{-1} \boldsymbol{\pi}_{i n}(\omega)
$$

On the other hand, the total energy of the response signal $\mathbf{V}^{\alpha}=\left(V_{1}^{\alpha}, \ldots, V_{r}^{\alpha}\right)$ is defined by the equation

$$
E_{t o t}^{\alpha}=\int_{\mathbb{R}}\left\|\mathbf{V}^{\alpha}(t)\right\|^{2} d t=\int_{B} \frac{1}{\pi}\left\|\mathbf{V}^{\alpha}(\omega)\right\|^{2} d \omega
$$

Introducing the random total spectral density function $s_{\alpha}^{v}$ of the random response velocity $\mathbf{V}^{\alpha}$ such that

$$
s_{\alpha}^{v}(\omega)=\frac{1}{\pi}\left\|\mathbf{V}^{\alpha}(\omega)\right\|^{2} .
$$

One then introduces the random spectral density function $\mathbf{s}^{v}$ of the random velocity responses $\mathbf{V}^{1}, \ldots, \mathbf{V}^{r}$ with values belonging to $\left(\mathbb{R}^{+}\right)^{r}$ such that

$$
\mathbf{s}^{v}(\omega)=\left(s_{1}^{v}(\omega), \ldots, s_{r}^{v}(\omega)\right) .
$$

Finally, one introduces the $(r \times r)$ real random matrix $\mathbf{H}(\omega)$ which can be define by

$$
\mathbf{H}_{\beta \alpha}(\omega)=\left|\mathbf{T}_{\beta \alpha}(\omega)\right|^{2} .
$$

From Eq. (12), it can then be deduced that $V_{\beta}^{\alpha}(\omega)=\mathbf{T}_{\beta \alpha}(\omega) f_{\alpha}^{\alpha}(\omega)$ without summation over $\alpha$. Thus, Eq. (24) yields $s_{\alpha}^{v}(\omega)=\frac{1}{\pi} \sum_{\beta=1}^{r}\left|\mathbf{T}_{\beta \alpha}(\omega)\right|^{2} f_{\alpha}^{\alpha}(\omega)^{2}$. Using Eqs. (19) and (26) one can obtain the equation $s_{\alpha}^{v}(\omega)=2 \sum_{\beta=1}^{r} \mathbf{H}_{\beta \alpha}(\omega) s_{\alpha}^{f}$ 
which can be rewritten as

$$
\mathbf{s}^{v}(\omega)=2 \mathbf{H}(\omega) \mathbf{s}^{f}(\omega) .
$$

Introducing the random local response power density function $\pi^{R}$ with values belonging to $\left(\mathbb{R}^{+}\right)^{r}$ such that

$$
\mathbf{s}^{v}(\omega)=\mathbf{Y}(\omega) \boldsymbol{\pi}^{R}(\omega)
$$

and substituting Eqs. (27) and (22) into Eq. (28), yields

$$
\boldsymbol{\pi}^{R}(\omega)=\mathbf{Y}(\omega)^{-1} \mathbf{H}(\omega) \mathbf{Y}(\omega)^{-1} \boldsymbol{\pi}_{i n}(\omega)
$$

From the right hand side of Eq. (29) one can define the $(r \times r)$ real full random matrix $\mathcal{E}(\omega)$ such as

$$
\mathcal{E}(\omega)=\mathbf{Y}(\omega)^{-1} \mathbf{H}(\omega) \mathbf{Y}(\omega)^{-1},
$$

it can easily be seen that Eq. (29) can be rewritten as

$$
\boldsymbol{\pi}^{R}(\omega)=\mathcal{E}(\omega) \boldsymbol{\pi}_{i n}(\omega)
$$

The two fundamental Eqs. (28) and (31) enable the calculation of $\mathbf{s}^{v}(\omega)$ as a function of $\boldsymbol{\pi}_{i n}(\omega)$ and using $\boldsymbol{\pi}^{R}(\omega)$. Consequently, the random matrix $\mathcal{E}(\omega)$ can be considered as a random dimensionless operator allowing the random local response power density function to be calculated as a function of the random input power density function. On the other hand, from Eqs. (27) and (30), the following fundamental equation can be deduced

$$
\mathbf{s}^{v}(\omega)=2 \mathbf{Y}(\omega) \mathcal{E}(\omega) \mathbf{Y}(\omega) \mathbf{s}^{f}(\omega)
$$

\section{Representation of the matrix-valued random FRF in the princi- pal directions of the mean local mobility}

It should be noted that the random equations defined by Eqs. (5) and (6) are expressed in the global coordinates system. In this section, one shows the representation of the FRF in a local coordinates system defined by the principal directions of the mean local mobility. Such a representation enables the type of dominant deformations to be analyzed with respect to the geometry. For instance, at a local point located in a thin shell of the structure, if the most important principal direction is perpendicular to the tangent plane of the shell, then the largest part of the energy of the response will be mainly associated with flexural deformations while if the most important principal direction belongs to the tangent plane, then the largest part of the energy will be mainly associated with membrane deformations. This is illustrated in 
Fig. 1 which shows two examples relative to the mobilities of the vibroacoustic system in the principal directions of the mean local mobility (in semi-log scale). One can notice that for some types of elements (flexible structural elements), the value of the mobility in the first principal direction of the mean local mobility is much higher than that in the two other principal directions over the frequency band of analysis. This is not the case for stiff structural elements where the values of the mobility in the three directions are similar. Thus, using the mobility analysis in the principal directions of the mean local mobility can also permit the different structural components to be classified. Moreover, one can notice that for Eq. (32) to be considered as a simplification of the spectral density function of the response, the mobility matrix should not be a full matrix. In the local coordinates defined by the principal directions of the mean local mobilities, the mobility matrix is a diagonal matrix by definition.
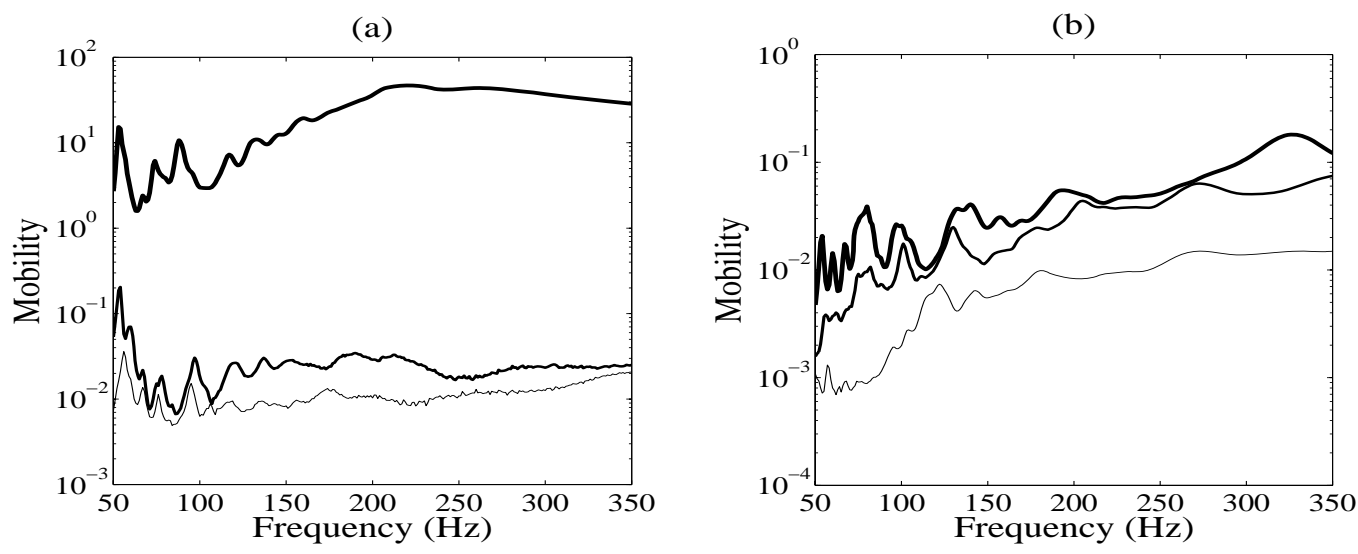

Figure 1. Mobility in the three principal local directions (first direction $X^{\ell}$ (thick line), second direction $Y^{\ell}$ (medium line), third direction $Z^{\ell}$ (thin line) at a point located in a flexible structural element (a) and at a point located in a stiff structural element (b).

\subsection{Introduction of local coordinates system defined by the principal directions of the mean local mobility}

Let $\mathbf{T}_{p}(\omega)$ be the random matrix with values in the set of all the symmetric complex $(3 \times 3)$ matrices and corresponding to the translational DOF's of the random FRF matrix $\mathbf{T}(\omega)$ at a given point $p$ of the structure (note that the rotational DOF's are not considered here). Since the structure is dissipative, it can be proven that $\Re \mathrm{e}\left\{\mathbf{T}_{p}(\omega)\right\}$ is a positive definite symmetric real random matrix. One then introduces the mean value $E\left\{\mathbf{T}_{p}(\omega)\right\}$ of the random matrix $\mathbf{T}_{p}(\omega)$ in which $E$ denotes the mathematical expectation. Let $\mathbb{T}_{p}(\omega)$ be the symmetric real $(3 \times 3)$ matrix such that $\mathbb{T}_{p}(\omega)=\Re e\left\{E\left\{\mathbf{T}_{p}(\omega)\right\}\right\}$. So, the symmetric real matrix $\mathbb{T}_{p}(\omega)$ is positive definite and can then be written as 
$\mathbb{T}_{p}(\omega)=\mathbb{X}_{p}(\omega) \Lambda(\omega) \mathbb{K}_{p}(\omega)^{T}$ in which $\mathbb{X}_{p}(\omega)$ is an orthogonal real $(3 \times 3)$ matrix (matrix of rotation in the three dimensional Euclidean space) made up of the eigenvectors of $\mathbb{T}_{p}(\omega)$ and where $\Lambda(\omega)$ is a diagonal matrix containing the positive eigenvalues associated to these eigenvectors. The local coordinates at this given point, defined by the principal directions of the mean local mobility, are such that $\mathbb{X}_{p}(\omega)$ maps the local coordinates into the global coordinates.

6.2 Representation of the local random FRF in the local coordinates system

The representation of the random matrix $\mathbf{T}_{p}(\omega)$ in the local coordinates attached to the given point $p$ and defined by the principal direction of the mean local mobility, is the random matrix denoted by $\mathbf{T}_{p}^{l o c}(\omega)$ and defined by

$$
\mathbf{T}_{p}^{l o c}(\omega)=\mathbb{X}_{p}(\omega)^{T} \mathbf{T}_{p}(\omega) \mathbb{X}_{p}(\omega)
$$

One can then define $\mathbf{H}_{p, i j}^{l o c}(\omega)=\left|\mathbf{T}_{p, i j}^{l o c}(\omega)\right|^{2}$ while $\mathbf{Y}_{p}^{l o c}(\omega)$ is constructed as in Eq. (13). Let $\mathbf{H}^{l o c}(\omega)$, $\mathbf{T}^{l o c}(\omega)$ and $\mathbf{Y}^{l o c}(\omega)$ be the matrices corresponding to the assemblage of these local matrices for all the local DOF's of the structure at points $p$ and for all the global DOF's of the acoustic cavity. One then obtains the following equation for the vibroacoustic system

$$
\mathcal{E}^{l o c}(\omega)=\mathbf{Y}^{l o c}(\omega)^{-1} \mathbf{H}^{l o c}(\omega) \mathbf{Y}^{l o c}(\omega)^{-1}
$$

All other equations of Section 5 still hold true in the local coordinates of the structure. Thus, these equations are going to be used in what follows with a subscript or a superscript loc to refer to values in these coordinates.

\section{Simplified statistical averaging model of the random matrix- valued FRF}

The complexity of the problem of the vibroacoustic analysis of industrial structures is induced by the large number of configurations which have to be studied. Consequently, simplified computational models have to be constructed from the full computational vibroacoustic model. In this Section, a simplified vibroacoustic model based on the energetic approach introduced in 5 and using statistical ensemble averaging, is presented. 


\subsection{Statistical averaging of the model parameters}

The mean matrix-valued FRF is calculated using all the realizations of the random matrix-valued FRF which is estimated using the Monte Carlo method after projection on the local coordinates (for the structure). The mean values are such that

$$
\underline{\mathbf{T}}^{l o c}(\omega)=E\left\{\mathbf{T}^{l o c}(\omega)\right\}
$$

and

$$
\underline{\mathcal{E}}^{l o c}(\omega)=E\left\{\mathcal{E}^{l o c}(\omega)\right\} .
$$

In this case, the mean local mobility is such that

$$
\underline{\mathbf{Y}}_{\alpha \beta}^{l o c}(\omega)=\left\{\begin{array}{cc}
\operatorname{Re}\left(\underline{\mathbf{T}}_{\alpha \alpha}^{l o c}(\omega)\right) & \text { if } \alpha=\beta \\
0 & \text { if } \alpha \neq \beta
\end{array} .\right.
$$

One can now define $\left(\underline{\mathbf{s}}_{l o c}^{v}(\omega)\right)^{r e f}$ as the exact value of the mathematical expectation of the random vector $\mathbf{s}^{v}(\omega)$ defined by Eq. (32) in the local coordinates and which is written as

$$
\underline{\mathbf{s}}_{l o c}^{v}(\omega)^{r e f}=2 E\left\{\mathbf{Y}^{l o c}(\omega) \mathcal{E}^{l o c}(\omega) \mathbf{Y}^{l o c}(\omega) \mathbf{s}_{l o c}^{f}(\omega)\right\} \quad,
$$

which is estimated using the Monte Carlo method.

\subsection{Construction of the simplified vibroacoustic model}

Let $J$ and $O$ be the set of excitation and observation DOF's respectively, such that $J=\left\{k_{q}, q=1, \ldots, \mu\right\}$ and $O=\left\{j_{p}, p=1, \ldots, \nu\right\}$, where $\mu$ and $\nu$ are the number of excitation and the number of observation DOF's respectively as illustrated in Fig. 2

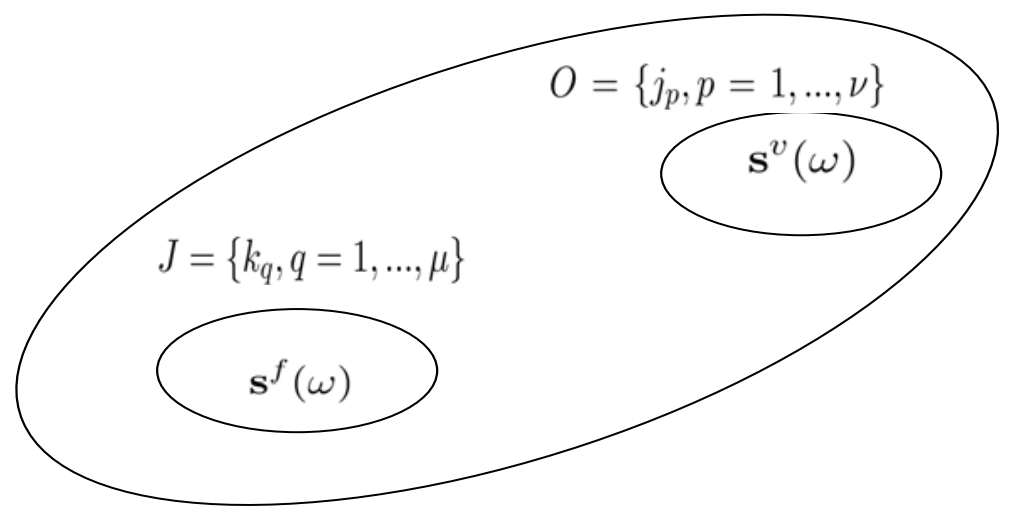

Figure 2. Schematic presentation of the sets of excitation and observation DOF's. 
Assuming that the excitation and observation DOF's $J$ and $O$ are sufficiently distant from each other, let $\underline{e}_{O J}(\omega)$ be the positive real number, such that for each $\omega$ in the frequency band $B$, one can write,

$$
\underline{\mathcal{E}}^{l o c}(\omega)_{j_{p} k_{q}} \simeq \underline{e}_{O J}(\omega)
$$

Using Eq. (39) and from Eq. (38), the following reasonable approximation can be deduced,

$$
\underline{\mathbf{s}}_{l o c}^{v}(\omega)_{j_{p}}^{a p p}=\underline{e}_{O J}(\omega) \underline{\mathbf{Y}}^{l o c}(\omega)_{j_{p} j_{p}} \underline{\pi}_{i n, J}^{l o c}
$$

where $\underline{\pi}_{i n, J}^{l o c}$ is the mean value of the random total input power relative to set $J$, which is defined by $\underline{\pi}_{i n, J}^{l o c}=\sum_{q=1}^{\mu} \underline{\pi}_{i n}^{l o c, q}$ in which $\underline{\pi}_{i n}^{l o c, q}$ is defined by Eqs. (20) and (21) expressed in the local coordinates system defined by the principal directions of the mean local mobility (for the structure). Clearly, the objective of this paper is to prove that such an approximation exists for complex vibroacoustic systems. It is however important to note that the spectral density function defined by Eq. (40) is an approximated value which is used to obtain an expression to calculate the positive real value $\underline{e}_{O J}(\omega)$. To calculate the value of $\underline{e}_{O J}(\omega)$ the reference value $\underline{\mathbf{s}}_{l o c}^{v}(\omega)^{\text {ref }}$ calculated using Eq. (38), (i.e. without any approximation) is used in the expression obtained from Eq. (40) such that

$$
\underline{e}_{O J}(\omega)=\frac{\sum_{p=1}^{\nu} \underline{\mathbf{s}}_{l o c}^{v}(\omega)_{j_{p}}^{r e f}}{\underline{\pi}_{i n, J}^{l o c} \sum_{p=1}^{\nu} \underline{\mathbf{Y}}^{l o c}(\omega)_{j_{p j} j_{p}}} .
$$

The value of the approximated mean vector-valued spectral density function of the output velocity can then be calculated by substituting $\underline{e}_{O J}(\omega)$, calculated with Eq. (41) into Eq. (40).

The value of the approximated mean vector-valued spectral density function of the output velocity can then be calculated by substituting $\underline{e}_{O J}(\omega)$ calculated using Eq. (41) into Eq. (40) in order to estimate the error induced by the approximation. The associated error due to the approximation can then be evaluated using two error functions. For the observation DOF's $j_{p}$ belonging to $O$ and for all $\omega$ belonging to the frequency band of analysis $B$, the first error function $\Delta(\omega)_{j_{p}}$ is defined by

$$
\Delta(\omega)_{j_{p}}=\left|\underline{\mathbf{s}}_{l o c}^{v}(\omega)_{j_{p}}^{r e f}-\underline{\mathbf{s}}_{l o c}^{v}(\omega)_{j_{p}}^{a p p}\right|
$$

which measures the accuracy of the calculation of $\underline{\mathbf{s}}_{l o c}^{v}(\omega)_{j_{p}}^{a p p}$ Eq. (40) with Eq. (41). The second error function is defined For all $j_{p}$ in $J$ and $k_{q}$ in $O$, and for all $\omega$ in $B$, the second error function $\varepsilon_{\mathcal{E}}(\omega)_{j_{p} k_{q}}$ such that

$$
\begin{aligned}
& \varepsilon_{\mathcal{E}}(\omega)_{j_{p} k_{q}}=\left|\mathrm{dB}(\omega)_{j_{p} k_{q}}-\mathrm{dB}(\omega)_{j_{p} k_{q}}^{a p p}\right|, \\
& \mathrm{dB}(\omega)_{j_{p} k_{q}}=10 \log _{10} \underline{\mathcal{E}}^{l o c}(\omega)_{j_{p} k_{q}} \quad, \quad \mathrm{~dB}(\omega)_{j_{p} k_{q}}^{a p p}=10 \log _{10} \mathbb{E}_{O J}(\omega)_{j_{p} k_{q}}^{a p p},
\end{aligned}
$$

where $\mathbb{E}_{O J}(\omega)_{j_{p} k_{q}}^{a p p}=e_{O J}(\omega)$. Eqs (43) and (44) measure the accuracy of the calculation of $\mathbb{E}_{O J}(\omega)_{j_{p} k_{q}}^{a p p}$. 


\section{Application to a complex vibroacoustic system}

The validation of the proposed energy method is performed on an automotive vehicle model. The mean vibroacoustic model consists of a non-trimmed vehicle structure and its internal acoustic cavity. The computational vibroacoustic model is made up of a finite element model of the structure and a finite element model of the internal acoustic cavity (see Fig. 3). The two finite element models are compatible on the coupling interface between the structure and the acoustic cavity. The finite element mesh of the structure is constituted of 1042851 DOF's and that of the acoustic cavity is constituted of 9157 DOF's. As mentioned earlier, only translational displacements of the structure are taken into account. Unit excitations forces are placed at each observation DOF's of the structure, while unit acoustic sources are placed at each observation DOF's in the acoustic cavity. So, the number of observation and excitation DOF's are equal. There are 12 excitation and observation points chosen in different zones of the internal acoustic cavity, and 28 points on the structure with a total of 96 DOF's. The excitation points on the structure correspond to: the points at which loads induced by the engine and the front suspension are applied; other points are chosen on the floor board, wind shield, roof and trunk board. This set of points is used to define the sets $\mathrm{O}$ and $\mathrm{J}$ in the following discussion. The vibroacoustic analysis is performed in the low- and medium-frequency band $B=[50,350] \mathrm{Hz}$.

In the reduced mean computational vibroacoustic model, the generalized structural damping matrix and the generalized acoustic damping matrix are generated as diagonal matrices whose diagonal terms are usually constructed with the damping rates of the modes. It is assumed that the damping rates are constant for all the elastic modes of the structure and are equal to 0.04 and are also constant for all the acoustic modes of the internal acoustic cavity and are equal to 0.1. For this reduced model and for the frequency band of analysis $\mathrm{B}$, the structure is represented by 1955 elastic modes and 3 rigid body translational modes $(n=1958)$, while the acoustic cavity is represented by 160 acoustic modes including the constant pressure acoustic mode $(m=160)$. These values of $n$ and $m$ are sufficiently high to get a good convergence of the deterministic and stochastic reduced models in the frequency band of analysis under consideration as proven in a previous work (see [14] [15]) for a similar computational vibroacoustic model. After constructing the matrices of the deterministic reduced mean computational vibroacoustic model as explained in 3 , the random matrices of the stochastic computational vibroacoustic model are constructed as explained in 4. Uncertainties are taken into account for the mass, damping and stiffness matrices of the structure and of the acoustic cavity as well as for the coupling matrix. The values of the dispersion parameters for these random matrices are those identified in previous work for a similar computational vibroacoustic model [14] [15]. The stochastic vibroacoustic equation is solved using the Monte Carlo method to obtain $n^{r}$ independent 
realizations.

(a)

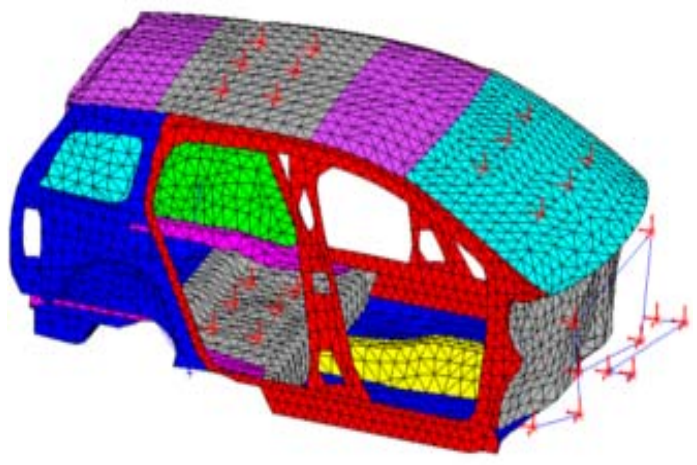

(b)

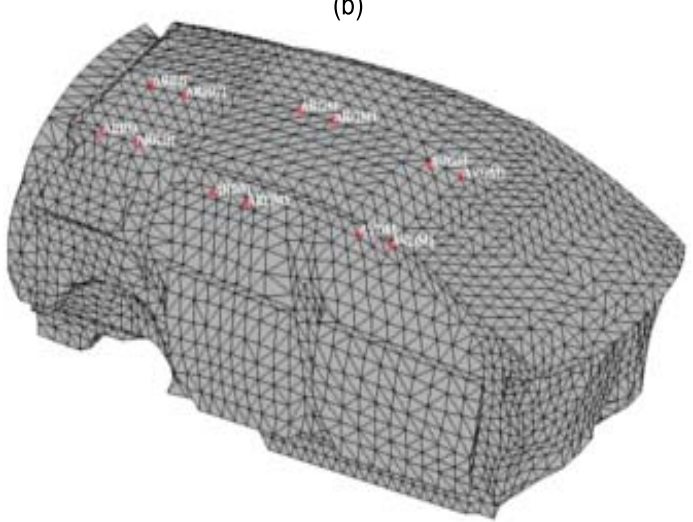

Figure 3. Finite element mesh of the structure (a) and of the acoustic cavity (b)

The mean-square convergence of the random solution is studied as a function of $n^{r}$ using the following function

$$
\operatorname{conv}^{H}\left(n^{r}\right)=\frac{1}{n^{r}} \sum_{\ell=1}^{n^{r}} \int_{B}\left\|\mathbf{Q}^{\mathbf{H}}\left(\omega ; \theta_{\ell}\right)\right\|^{2} d \omega
$$

in which $H$ stands for the letter $s$ designating the structure and for the letter $f$ designating the acoustic fluid, and where $\mathbf{Q}^{\mathbf{H}}\left(\omega ; \theta_{1}\right), \ldots, \mathbf{Q}^{\mathbf{H}}\left(\omega ; \theta_{n^{r}}\right)$ are the independent realizations of the vector-valued random variable $\mathbf{Q}^{\mathbf{H}}(\omega)$ constructed with Eq. (6). Fig. 4 shows the graphs of $\operatorname{conv}^{s}\left(n^{r}\right)$ and $\operatorname{conv}^{f}\left(n^{r}\right)$, for the structure and for the acoustic fluid, respectively, as a function of $n^{r}$.
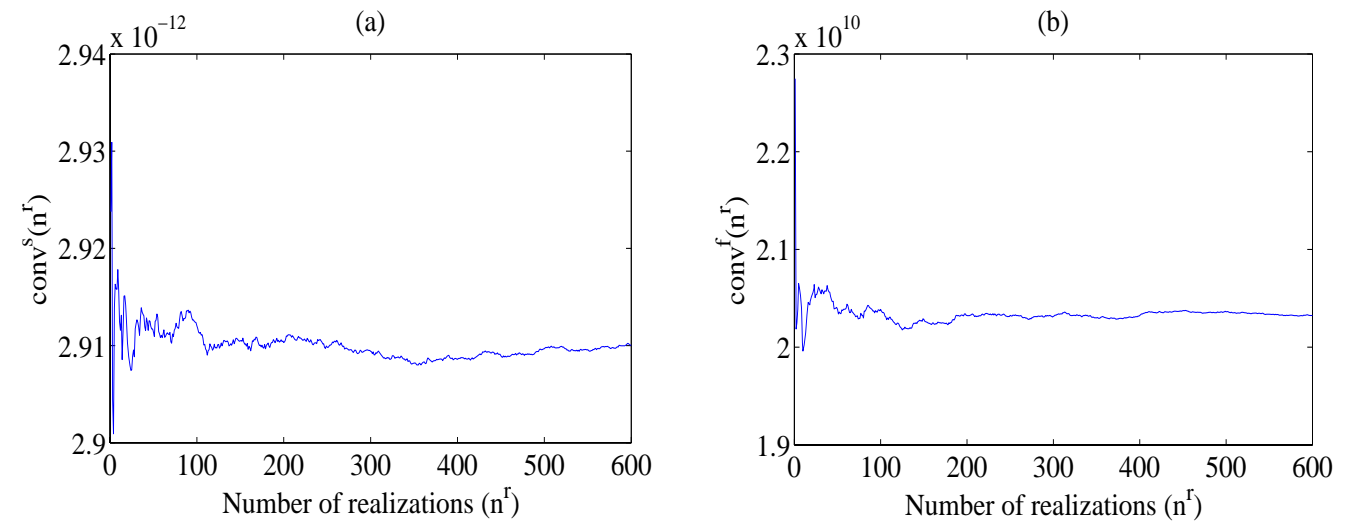

Figure 4. Graphs of $\operatorname{conv} v^{s}\left(n^{r}\right)$ for the structure (a) and of $c o n v^{f}\left(n^{r}\right)$ for the acoustic fluid (b) as a function of $n^{r}$.

Fig. 4 shows that convergence occurs at about 550 realizations for the structure and at about 400 realizations for the acoustic fluid. Thus, all the results 
presented have been computed using 600 realizations to ensure the convergence for both the structure and the acoustic cavity. The local coordinates systems defined by the principal directions of the mean local mobilities are calculated as explained in Section 6.1. The local coordinates are then denoted by $\left(X^{\ell}, Y^{\ell}, Z^{\ell}\right)$. Each realization $\mathbf{T}_{p}^{l o c}(\omega, \theta)$ and $\mathcal{E}^{l o c}(\omega, \theta)$ are calculated using Eqs. (33) and (34). The mean values $\underline{\mathbf{T}}^{l o c}(\omega)$ and $\underline{\mathcal{E}}^{l o c}(\omega)$ are calculated using Eqs. (35) and (36). The confidence regions of $\mathbf{T}_{p}^{l o c}(\omega)$ and $\mathcal{E}^{l o c}(\omega)$ are constructed using the quantiles method (see[20]) for a probability level of 0.95 . Figs. 5, 6 and 7 show the mean values $\underline{\mathbf{T}}^{l o c}(\omega)$ and $\underline{\mathcal{E}}^{l o c}(\omega)$ of $\mathbf{T}^{l o c}(\omega)$ and $\mathcal{E}^{l o c}(\omega)$ for different excitation and observation points.

(a)

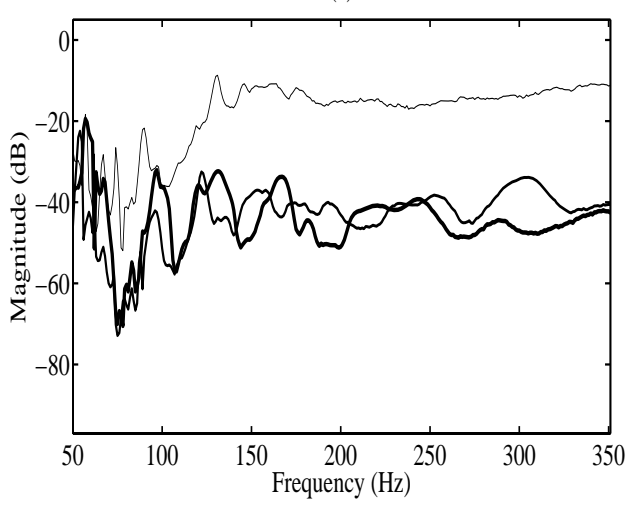

(b)

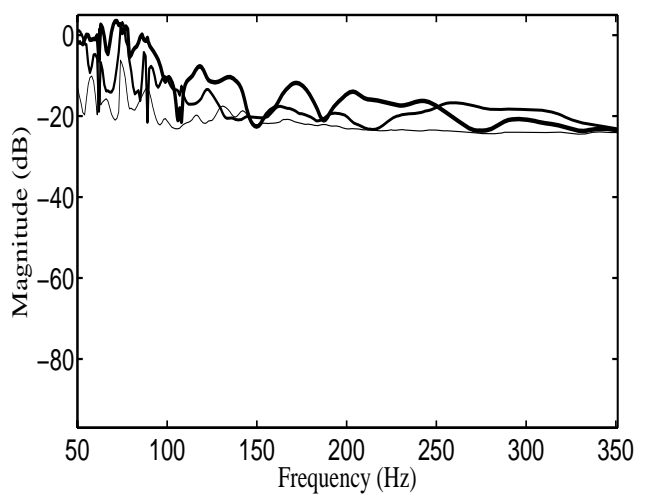

Figure 5. Graphs of $\underline{\mathbf{T}}^{l o c}(\omega)$ (a) and of $\underline{\mathcal{E}}^{l o c}(\omega)$ (b) for the structure input - structure output FRF as a function of the frequency. The structure output is the structure velocity in direction $X^{\ell}$. The three structure inputs are the structural forces applied in the three local principal directions $X^{\ell}$ (thin line), $Y^{\ell}$ (medium line), $Z^{\ell}$ (thick line).

(a)

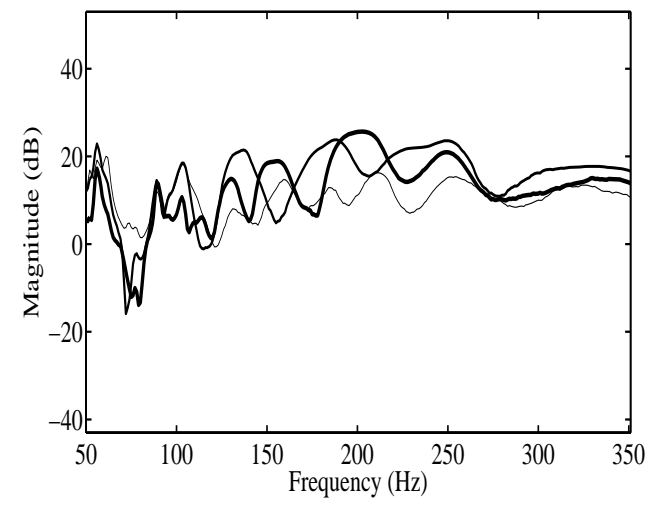

(b)

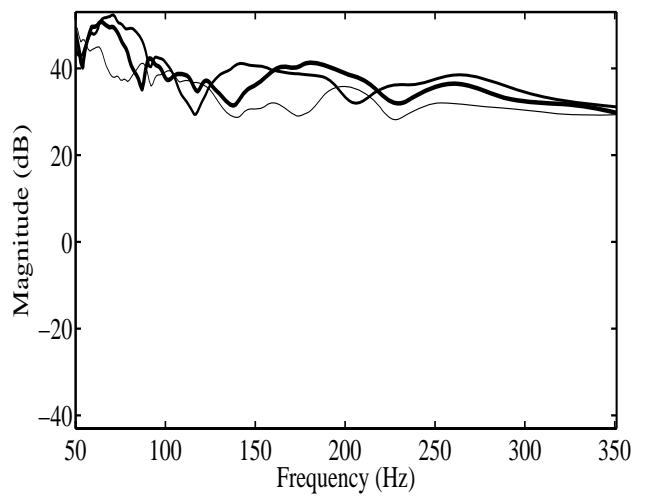

Figure 6. Graphs of $\underline{\mathbf{T}}^{l o c}(\omega)$ (a) and of $\underline{\mathcal{E}}^{l o c}(\omega)$ (b) for three acoustic input - acoustic output FRF as a function of the frequency. Each corresponds to a given excitation point and a given observation point inside the acoustic cavity. 
(a)

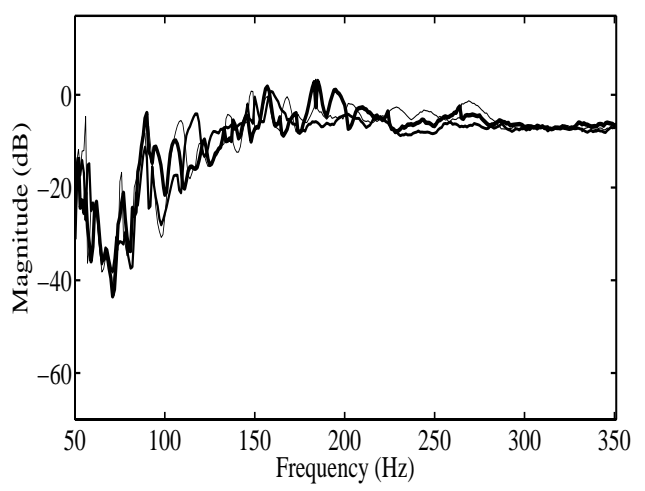

(b)

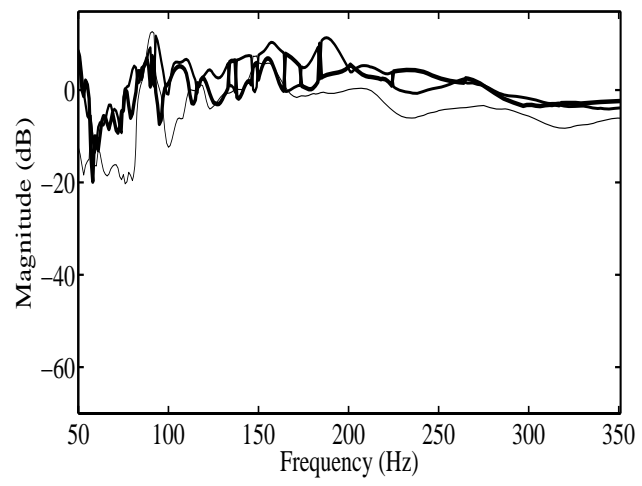

Figure 7. Graphs of $\underline{\mathbf{T}}^{l o c}(\omega)$ (a) and of $\underline{\mathcal{E}}^{l o c}(\omega)$ (b) for the structure input - acoustic output FRF as a function of the frequency. The acoustic output is the pressure at a given point inside the acoustic cavity. The three structure inputs are the structural forces applied in the three local principal directions $X^{\ell}$ (thin line), $Y^{\ell}$ (medium line), $Z^{\ell}$ (thick line).

From 5 to 7 , one can notice that the variation in magnitude, as a function of frequency, of $\underline{\mathcal{E}}^{l o c}(\omega)$ is less than that of $\underline{\mathbf{T}}^{l o c}(\omega)$. Moreover, from Figs. 5 and 7 it has been noticed that when fixing an observation point and changing the direction of excitation among the three local principal directions or vice versa, $\underline{\mathcal{E}}^{l o c}(\omega)$ seems to undergo less changes than $\underline{\mathbf{T}}^{l o c}(\omega)$. It can also be seen that the three curves corresponding to the excitations in the three directions of the normalized FRF tend towards an asymptotic value starting at about $300 \mathrm{~Hz}$. This illustrates the independency of the normalized FRF from the direction of excitation. The independency of the normalized FRF from the direction of observation was also verified. This considerably reduces the size of the problem to be analyzed. Figs. 8 to 10 show the confidence regions for $\mathbf{T}^{l o c}(\omega)$ and $\mathcal{E}^{l o c}(\omega)$ corresponding to different excitation-observation combinations.
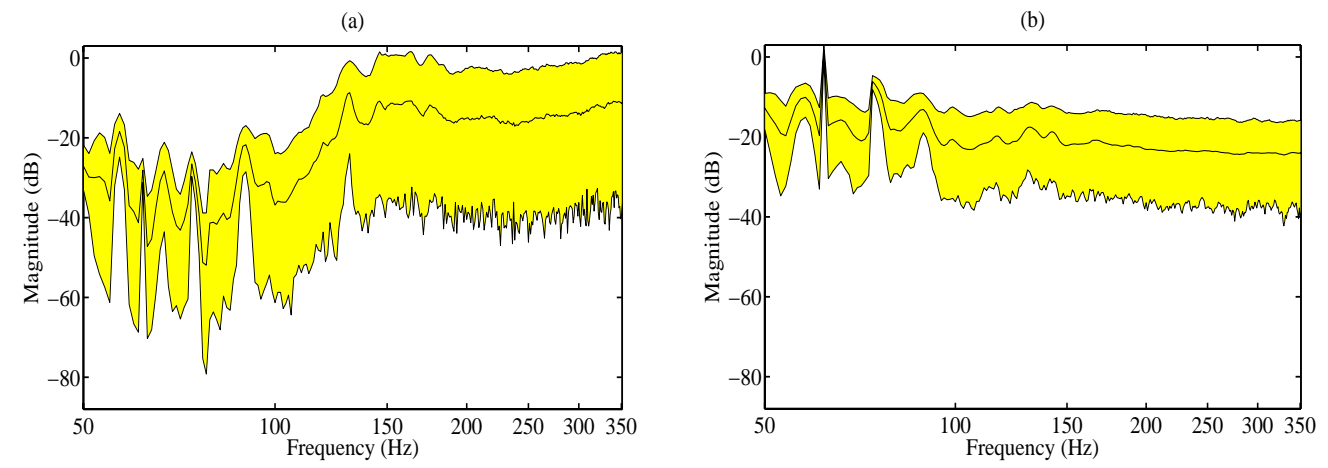

Figure 8. Confidence regions of $\mathbf{T}^{l o c}(\omega)$ (a) and $\mathcal{E}^{l o c}(\omega)$ (b). Excitation is a force applied to a given point on the structure in the $X^{\ell}$ direction. Observation is the velocity in another point of the structure in the $X^{\ell}$ direction. Medium line is the mean value. Upper and lower lines delimit the confidence region. 

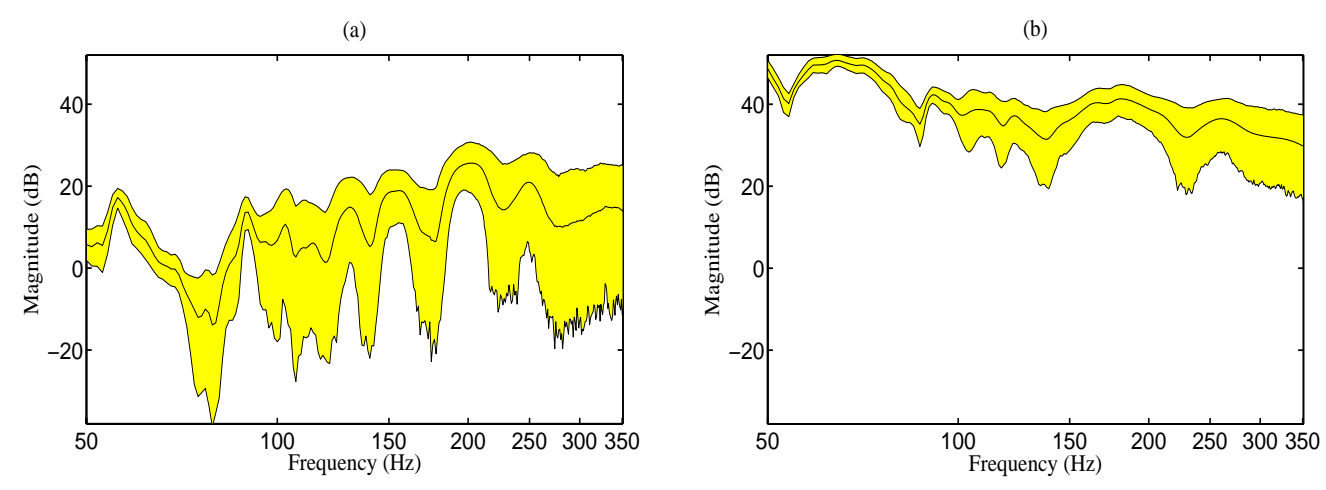

Figure 9. Confidence regions of $\mathbf{T}^{l o c}(\omega)$ (a) and $\mathcal{E}^{l o c}(\omega)$ (b). Excitation is an acoustic pressure applied to a given point in the acoustic cavity. Observation is the acoustic pressure observed in another point of the acoustic cavity. Medium line is the mean value. Upper and lower lines delimit the confidence region.
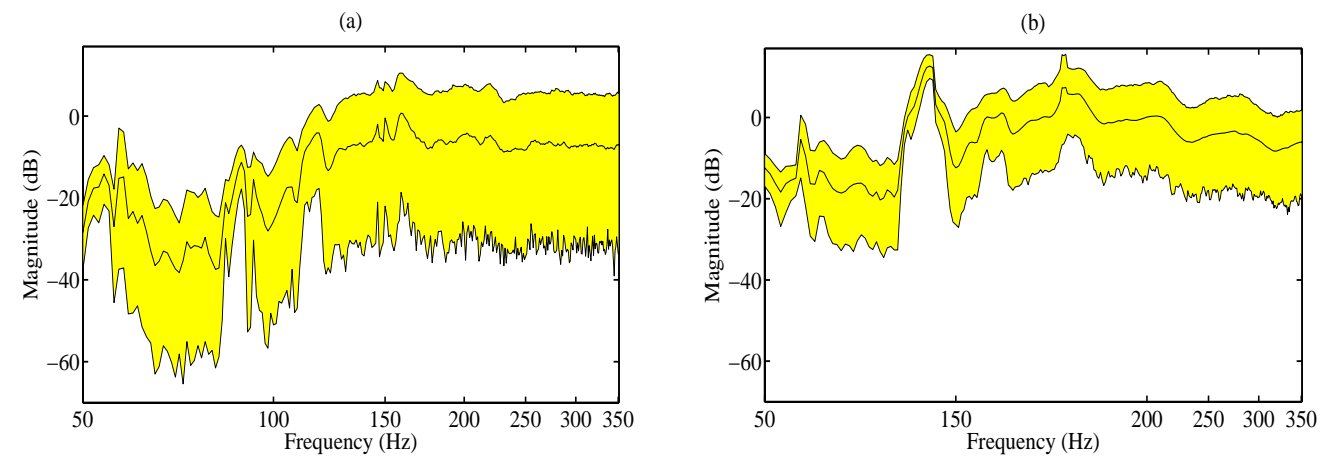

Figure 10. Confidence regions of $\mathbf{T}^{l o c}(\omega)$ (a) and $\mathcal{E}^{l o c}(\omega)$ (b). Excitation is a force applied to a given point on the structure in the $X^{\ell}$ direction. Observation is the acoustic pressure observed in a point of the acoustic cavity. Medium line is the mean value. Upper and lower lines delimit the confidence region.

From From Figs. 8 to 10, it can be noted that the confidence region around the mean value $\underline{\mathcal{E}}^{l o c}(\omega)$ is smaller than that around the mean value $\underline{\mathbf{T}}^{l o c}(\omega)$. In other words, using $\mathcal{E}^{l o c}(\omega)$ as a representation of the FRF yields less dispersion in the results than using $\mathbf{T}^{l o c}(\omega)$. This decrease in the dispersion values increases the predictability of the model and makes it more robust regarding uncertainties. So, the normalized FRF is a more reliable parameter than the usual FRF. The observations mentioned above regarding the response of the vibroacoutic system made it interesting to observe the behavior of the matrix of the normalized FRF for all degrees of freedom as a function of frequency. This is illustrated in Fig. 11 which shows the color plots of the matrix $\underline{\mathcal{E}}_{l o c}(\omega)$ at frequencies $70 \mathrm{~Hz}, 170 \mathrm{~Hz}, 270 \mathrm{~Hz}$ and $350 \mathrm{~Hz}$. 
(a)

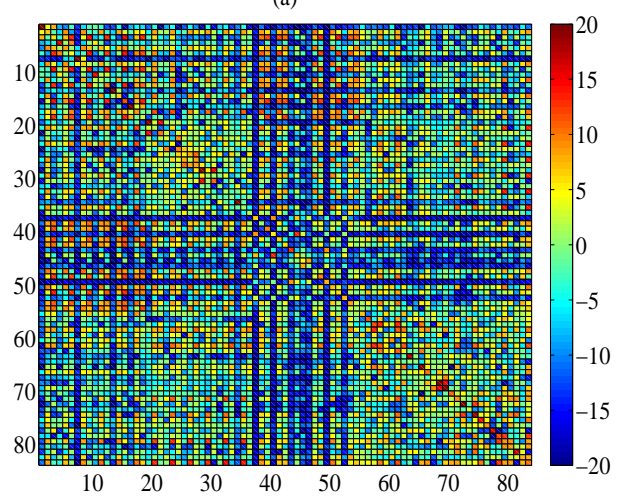

(c)

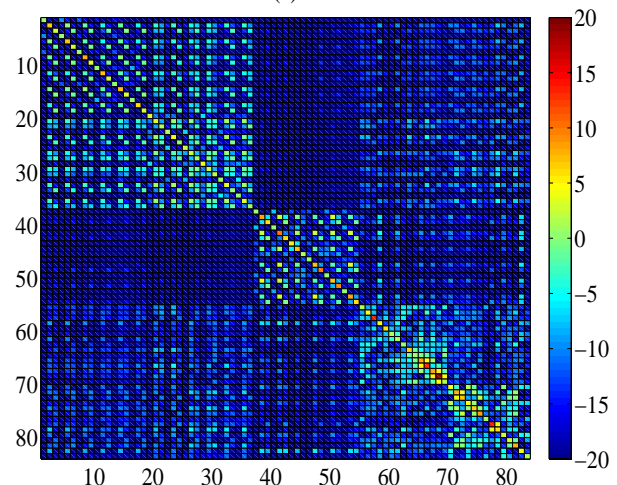

(b)

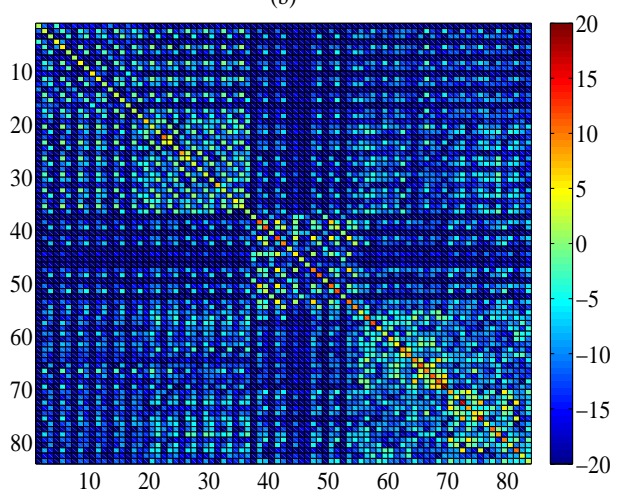

(d)

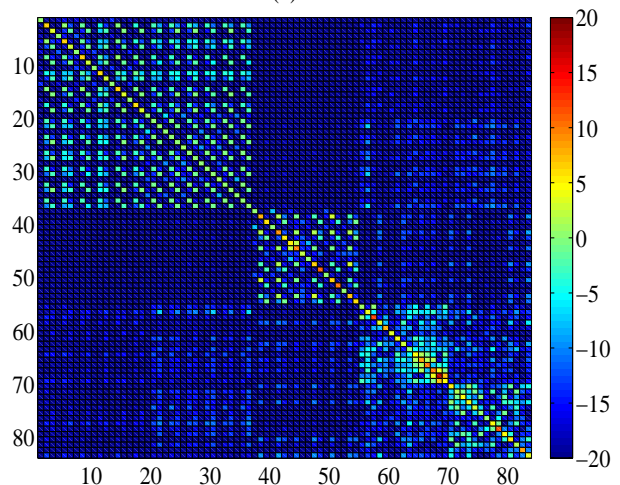

Figure 11. Color plot of $\underline{\mathcal{E}}_{l o c}(\omega)$ at $70 \mathrm{~Hz}(\mathrm{a}), 170 \mathrm{~Hz}(\mathrm{~b}), 270 \mathrm{~Hz}(\mathrm{c})$ and $350 \mathrm{~Hz}(\mathrm{~d})$.

From Fig. (11), it can be seen that starting at about $170 \mathrm{~Hz}$ the color plot of the matrix takes a block diagonal form. The blocks on the diagonal correspond to different parts of the structure. Extra-diagonal blocks can also be observed. These extra-diagonal blocks represent coupling between different parts of the structure. At higher frequencies the color representation of the matrix gets stable which confirms the results shown in Fig. Fig. 5(b) concerning the convergence of the response towards an asymptotic value. Moreover, away from the block diagonal terms, several zones (extra-diagonal blocks) have a uniform color indicating constant values. This result also confirms the observations made on Fig. Fig. 5(b) concerning the independency of the value of $\underline{\mathcal{E}}_{l o c}(\omega)$ from the excitation and observation directions. Moreover, these constant value zones group several points belonging to different parts of the structure which means that these zones have the same behavior regarding the imposed excitation. Taking into account the previous comments, it is now important to show the error matrix $\varepsilon_{\mathcal{E}}(\omega)$ to better understand the domain of validity of the approximation defined using the positive real value $\underline{e}_{O J}(\omega)$. $\underline{e}_{O J}(\omega)$ is calculated using Eq. (41). The $\left(n_{\mu} \times n_{\nu}\right)$ matrix $\varepsilon_{\mathcal{E}}(\omega)$ is then calculated using Fig. 12 . Fig. 12 shows the color plots of the $\left(n_{\mu} \times n_{\nu}\right)$ matrix $\varepsilon_{\mathcal{E}}(\omega)$ at frequencies 70 $\mathrm{Hz}, 170 \mathrm{~Hz}, 270 \mathrm{~Hz}$ and $350 \mathrm{~Hz}$. 
(a)

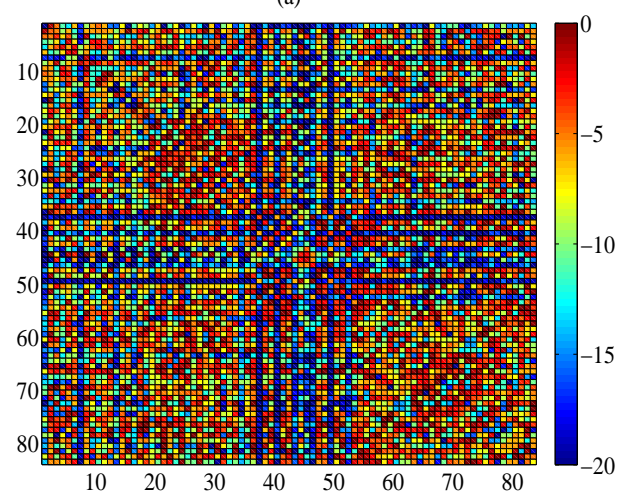

(c)

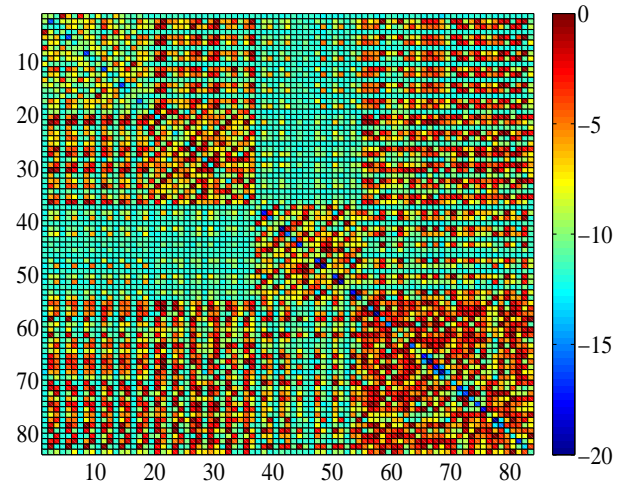

(b)

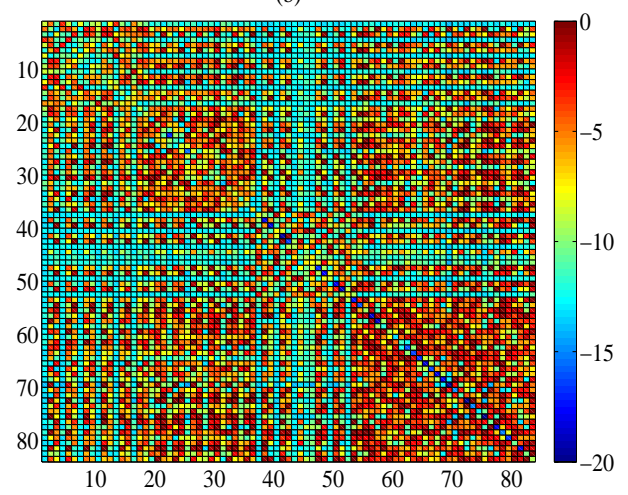

(d)

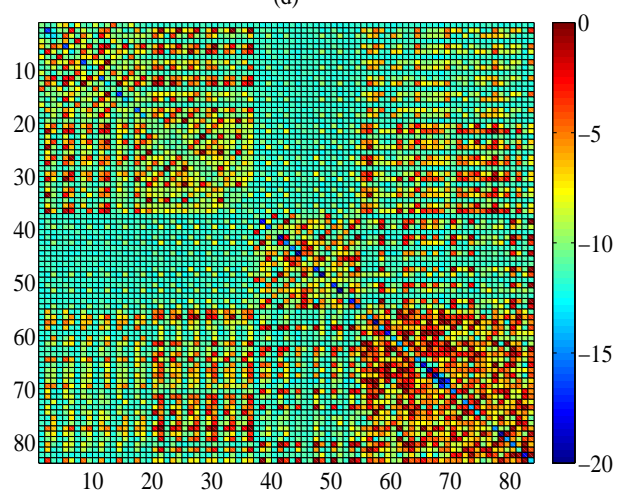

Figure 12. Color plots of $\varepsilon_{\mathcal{E}^{(}}(\omega)$ at $70 \mathrm{~Hz}(\mathrm{a}), 170 \mathrm{~Hz}(\mathrm{~b}), 270 \mathrm{~Hz}$ (c) and $350 \mathrm{~Hz}$ (d).

From Fig. 12, it can be seen that, at low frequency the error between $\mathbb{E}_{O J}(\omega)$ and $\underline{\mathcal{E}}_{l o c}(\omega)$ is high at most of the matrix elements. At higher frequencies the error decreases away from the diagonal terms, that is to say when the excitation and observation points are far enough from each others confirming the starting hypothesis used for the construction of the simplified model. Such a behavior seems to hold true for frequencies higher than $170 \mathrm{~Hz}$. Again each block on the diagonal of the matrix corresponds to the DOF's located on the same part of the structure. We can then conclude that the positive real value $\underline{e}_{O J}(\omega)$ can be used to approximate $\underline{\mathcal{E}}_{l o c}(\omega)_{j_{p} k_{q}}$ see Eq. (39) between zones of the structure for which the corresponding error function is small.

\section{Conclusion}

An energy density field approach for complex vibroacoustic systems has been presented and validated in the low- and medium-frequency ranges. This method is based on the introduction of a dimensionless energy density function and its averaging over a set of random vibroacoustic systems, deduced from the 
deterministic nominal computational vibroacoustic model, obtained using a probabilistic model of uncertainties. The energy density field enables an approximation of the FRF to be introduced. The concept of local coordinates system defined by the principal directions of the mean local mobility was also presented. This coordinates system enables the type of dominant deformations to be analyzed and prevent the loss of information that may be associated with the elimination of coupling terms of the mobility matrix (diagonal matrix). A Simplified model is constructed based on this energy-density field approach with the hypothesis that the excitation points and the observation points are sufficiently distant from each others. The response of this simplified model is shown to be less dispersed than the usual FRF and thus provides more reliable and robust results. Two error functions are introduced in order to estimate the error due to the presented approximation. These error functions also enable the automatic identification of zones for which the approximation holds true. The proposed method and the simplified model have been validated on a sufficiently complex vibroacoustic system (Automotive vehicle). A straight forward extension of this approach and the model simplification is an automatic substructuring technique. This technique is particularly useful in the context of concurrent engineering. It enables time reduction of the conception process by providing the response of a complete zone rather than discrete DOF's and by providing a powerful tool for the predictions of low- and medium-frequency vibroacoustic behavior of the complex structure.

\section{References}

[1] C. Soize, Maximum entropy approach for modeling random uncertainties in transient elastodynamics. Journal of Acoustical Society of America, 109(5), pp. 1979-1996, 2001.

[2] C. Soize, A comprehensive overview of a non-parametric probabilistic approach of model uncertainties for predictive models in structural dynamics, Journal of sound and vibration, 288, pp. 623-652, 2005.

[3] C. Soize, Random matrix theory for modeling uncertainties in computational mechanics. Comput. Methods Appl. Mech. Engrg., 194, pp. 13331366, 2005.

[4] R.H. Lyon, Statistical Energy Analysis of Dynamical Systems, MIT Press, San Diego, 1975.

[5] R.H. Lyon, Statistical Theory and application of statistical energy analysis, 2nd edition, Butterworth-Heinemann, Boston, MA, 1995.

[6] F.J. Fahy, A.D. Mohammed, A study of uncertainty in applications of SEA to coupled beam and plate systems, partI: Computational experiments, Journal of Sound and Vibration, 158, pp. 45-67, 1992.

[7] R.S. Langley, P. Bremner, A hybrid method for the vibration analysis of complex structural-acoustic systems, The Journal of the Acoustical Soci- 
ety Of America, 105(3), pp. 1657-1671, 1999.

[8] L. Maxit, J.-L. Guyader, Extension of SEA model to subsystems with non-uniform modal energy distribution, Journal of Sound and Vibration, 265, pp. 337-358, 2003.

[9] R.S. Langley, A.W.M. Brown, The ensemble statistics of the bandavaraged energy of random system, Journal of Sound and Vibration, 275, pp. 847-857, 2004.

[10] R.S. Langley, V. Cotoni, Response variance prediction in the statistical energy analysis of built up systems, The Journal of the Acoustical Society Of America, 115(2), pp. 706-718, 2004.

[11] P.J. Shorter, R.S. Langley, Vibroacoustic analysis of complex systems, Journal of Sound and Vibration, 288, pp. 669-699, 2005.

[12] V. Cotoni, R.S. Langley, M.R.F. Kinder, Numerical and experimental validation of variance prediction in the statistical energy analysis of builtup systems, Journal of Sound and Vibration, 288, pp. 701-728, 2005.

[13] N. Totaro, J.L. Guyader, SEA substructuring using cluster analysis: The MIR index, Journal of Sound and Vibration, 290, pp. 264-289, 2006.

[14] J.F Durand, C. Soize, L. Gagliardini, Structural-acoustic modeling of automotive vehicles in presence of uncertainties and experimental identification and validation. The Journal of the Acoustical Society of America, 124(3), pp. 1513-1525, 2008.

[15] J.F. Durand, Structural-acoustic modeling of automotive vehicles in presence of modeling uncertainties and experimental validation. Phd Thesis (in french), Université de Marne-la-Vallée, 2007.

[16] G. Orefice, C. Cacciolati, J.L. Guyader, The energy mobility, Journal of Sound and Vibration, 254(2), (2002), pp. 269-295, 2002.

[17] R. Ohayon and C. Soize, Structural Acoustics and Vibrations, Academic Press, San Diego, 1998.

[18] L. Gagliardini, G. Borello, Virtual SEA: Towards an industrial process. SAE International, 2007-01-2302, 2007.

[19] Y.K. Koh, R.G. White, Analysis and control of the vibrational power transmission to machiery supporting structures subjected to a multiexcitation system, partI: Driving point mobility matrix of beams and rectangular plates. Journal of Sound and Vibration, 196(4), pp. 469-493, 1996.

[20] R.J. Serfling, Approximation Theorems of Mathematical Statistics, Wiley, New york, 1980. 